\title{
Managements of 13 emergency cardiac surgeries under COVID-19 pandemic in a Sentinel Hospital
}

\author{
Xingjian Hü^, Yin Wang", Jiahong Xia, Nianguo Dong, Jiawei Shi \\ Department of Cardiovascular Surgery, Wuhan Union Hospital, Tongji Medical College, Huazhong University of Science and Technology, Wuhan, \\ China \\ Contributions: (I) Conception and design: X Hu, J Shi; (II) Administrative support: J Xia, N Dong; (III) Provision of study materials or patients: N \\ Dong, J Shi; (IV) Collection and assembly of data: X Hu, Y Wang; (V) Data analysis and interpretation: X Hu, Y Wang; (VI) Manuscript writing: All \\ authors; (VII) Final approval of manuscript: All authors. \\ "These authors contributed equally to this work as co-first authors. \\ Correspondence to: Nianguo Dong, MD, PhD; Jiawei Shi, MD, PhD. Department of Cardiovascular Surgery, Wuhan Union Hospital, Tongji Medical \\ College, Huazhong University of Science and Technology, Jiefang Avenue 1277\#, Wuhan 430000, China. \\ Email: dongnianguo@hotmail.com; shijiawei@21cn.com.
}

Background: The pneumonia outbreak caused by the 2019 novel coronavirus disease (COVID-19) creates many challenges for the healthcare sector. Currently, little is known of how the pandemic has impacted patients with cardiovascular disease. The primary focus of this study was to determine whether emergency cardiovascular surgeries can be carried out safely during the COVID-19 pandemic.

Methods: Between 17 January 2020 and 11 February 2020, 13 patients were admitted to Wuhan Union Hospital for emergency cardiovascular surgery. During this time, Wuhan was a COVID-19 epicenter, and Wuhan Union Hospital is a sentinel hospital located in this area. These patients' epidemiological histories, clinical records, laboratory assessments, imaging findings, and surgical outcomes were retrospectively reviewed. Throat swabs were collected from some patients preoperatively and all patients postoperatively for reverse transcription polymerase chain reaction (RT-PCR) testing to determine whether these patients had COVID-19.

Results: This cohort included 5 cases of acute aortic dissection, 3 cases of congenital heart disease, 2 cases of dilated cardiomyopathy with end-stage heart failure, 1 case of aortocoronary fistula that had undergone previous surgery, 1 case of subacute infective endocarditis with cerebral infarction, and 1 case of multivessel coronary disease. Six patients were suspected COVID-19 cases (46.2\%). There were no confirmed COVID-19 cases in this cohort. None of the patients in this cohort died and none developed severe acute respiratory syndrome, renal failure, or septic shock after surgery. No cross-infection occurred with other patients or medical staff who came into close contact with this cohort.

Conclusions: Emergency surgery is crucial and unavoidable for many patients with acute and severe cardiovascular disease, regardless of the pandemic. Our study indicates that, with adequate preparation and the provision of appropriate treatment, satisfactory outcomes can be achieved for such patients.

Keywords: Clinical experience; 2019 novel coronavirus disease (COVID-19); emergency cardiovascular surgery

Submitted Apr 12, 2020. Accepted for publication Sep 23, 2020.

doi: $10.21037 /$ jtd-20-1649

View this article at: http://dx.doi.org/10.21037/jtd-20-1649

$\wedge$ ORCID: 0000-0002-8554-6138. 


\section{Introduction}

In early December 2019, an outbreak of pneumonia of unknown origin was first reported in Wuhan, China. Since, cases of respiratory illness have spread worldwide, causing a serious pandemic (1). From the clinical data and information accumulated, a novel beta-coronavirus known as severe acute respiratory syndrome coronavirus 2 (SARS-CoV-2) has been confirmed as the causative agent (2). SARS-CoV-2 is highly infectious and manifests as the viral pneumonia now known as coronavirus disease 2019 (COVID-19). This virus can transmit from person to person via respiratory droplets or direct contact. As of 26 February 2020, 78,196 COVID-19 cases had been confirmed in China (including Hong Kong, Macao, and Taiwan), with 2,718 confirmed deaths, and there were 3,078 confirmed cases and 51 confirmed deaths across more than 30 other countries $(3,4)$. This ongoing outbreak has been declared a Public Health Emergency of International Concern (PHEIC) by the World Health Organization (WHO).

Cardiovascular disease frequently occurs during the change of season from winter to spring, COVID-19 was also prevalent in China during this period. Additionally, some research suggests that SARS-CoV-2 might affect the cardiovascular system via angiotensin converting enzyme 2 (ACE2) receptors $(5,6)$. Therefore, COVID-19 patients with underlying cardiovascular disease might have an increased risk of developing acute symptoms of the disease and deterioration. Emergency surgery is crucial and unavoidable for patients with acute aortic dissection, acute myocardial infarction, and end-stage heart failure. However, neither the WHO Interim Guidelines nor the Novel Coronavirus Pneumonia Prevention and Control Program published by the National Health Commission of China has offered specific recommendations for the management of COVID-19 patients who require emergency cardiovascular surgery $(7,8)$. In this study, we retrospectively analyzed the clinical data and therapeutic outcomes of suspected COVID-19 patients who underwent emergency cardiovascular operation in the Department of Cardiovascular Surgery at Wuhan Union Hospital. Our aim was to summarize the effective management of these cases. This article is presented in accordance with the Strengthening the Reporting of Observational Studies in Epidemiology (STROBE) Statement reporting checklist (available at http://dx.doi.org/10.21037/jtd-20-1649).

\section{Methods}

A total of 20 consecutive patients with acute and severe cardiovascular disease were admitted to Wuhan Union Hospital between 17 January 2020 and 11 February 2020. The clinical data and outcomes of this cohort were retrospectively reviewed. The epidemiological histories, clinical symptoms, comorbidities, imaging indexes, laboratory test results, surgical features, and clinical outcomes of these patients were obtained from their electronic medical records. Laboratory assessments comprised blood tests [complete blood count (CBC), total bilirubin, alanine aminotransferase (ALT), and creatinine, etc.], cardiac enzyme tests [lactate dehydrogenase (LDH), creatine kinase-MB (CK-MB), aspartate aminotransferase (AST), troponin I (TnI), C-reactive protein (CRP), and procalcitonin, etc.], and routine respiratory pathogen tests. Imaging methods included chest X-rays, computed tomography (CT), and echocardiography. All data were reviewed by two analysts (XH and YW) to verify their accuracy.

Diagnoses of COVID-19 pneumonia at Wuhan Union Hospital are based on the Novel Coronavirus Pneumonia Prevention and Control Program ( $6^{\text {th }}$ edition), published by the National Health Commission of China (8). Suspected cases are identified as patients with an epidemiological history of COVID-19 who also demonstrate at least two of the following requirements: typical respiratory symptoms or fever; abnormal hemogram results; or positive chest CT results. Suspected cases are confirmed according to positive reverse transcription polymerase chain reaction (RT-PCR) results; the RT-PCR is performed using respiratory tract swabs.

This study was conducted in accordance with the Declaration of Helsinki (as revised in 2013), and approved by the Medical Ethical Committee of Wuhan Union Hospital of Huazhong University of Science and Technology \{[2020]IRS(S229)\}. Written informed consent was obtained from all study participants or their legal guardians; patient anonymity was assured throughout the research process.

\section{Statistical analysis}

Continuous variables were expressed as mean \pm standard deviation. Categorical variables were summarized as the 

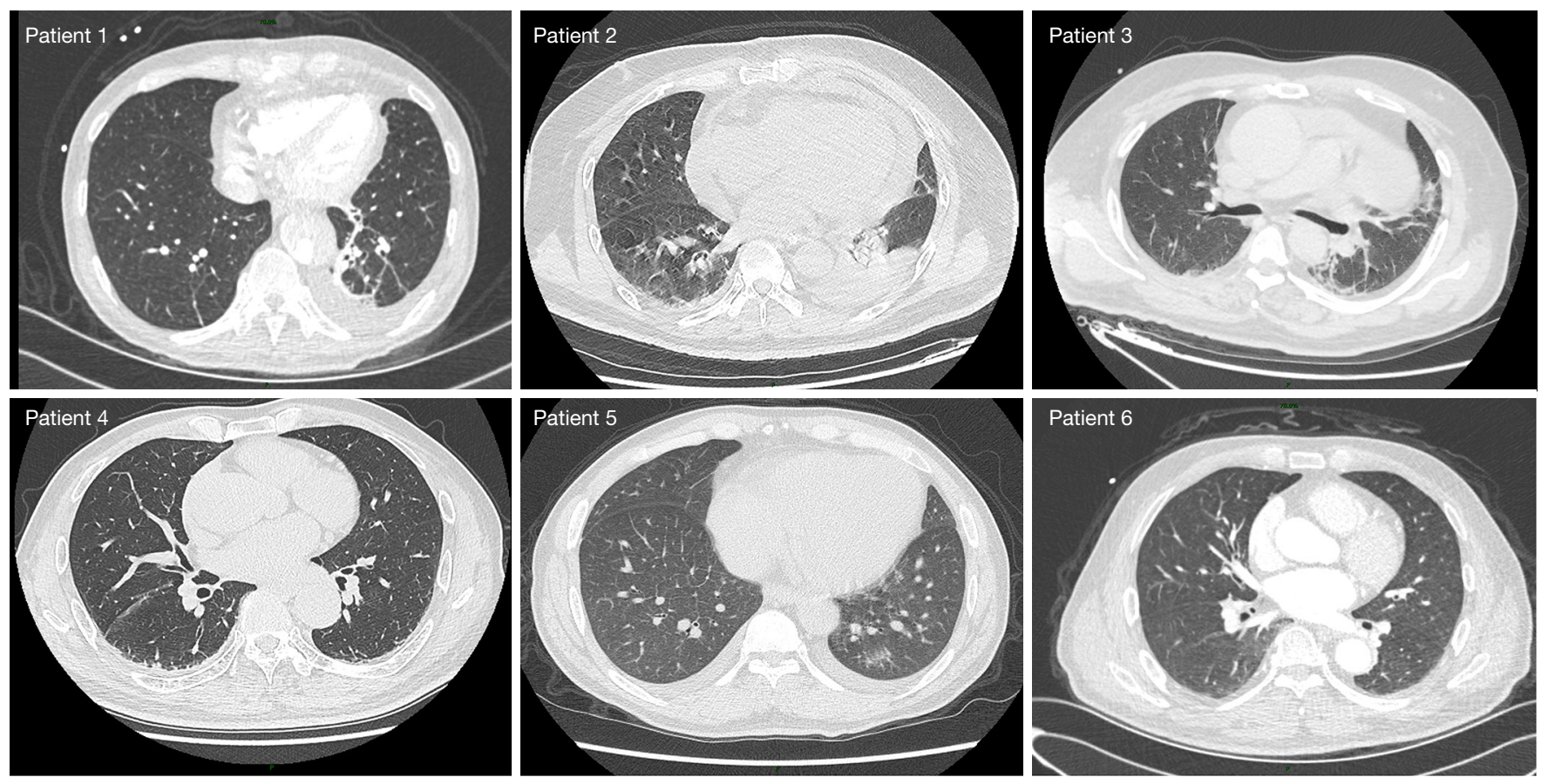

Figure 1 Representative patient chest computed tomography image depicting changes that can occur with pneumonia.

counts and percentages for each category.

\section{Results}

Of the 20 patients admitted between 17 January 2020 and 11 February 2020, 7 non-emergency patients were treated with conservative medication and advised to wait until after the pandemic to receive surgery. The remaining 13 patients were diagnosed with acute and severe cardiovascular disease and underwent emergency surgery. Of these patients, 5 had acute aortic dissection, 3 had congenital heart disease with severe pulmonary hypertension or heart failure, 2 had dilated cardiomyopathy with end-stage heart failure, 1 had aortocoronary fistula with heart failure following a previous operation, 1 had subacute infective endocarditis with cerebral infarction, and 1 had multivessel coronary disease.

\section{Demographic and clinical characteristics}

This cohort ranged in age from 1 month to 64 years and was predominantly male $(84.6 \% ; 11 / 13)$. Only 5 (38.7\%) of the patients had a history of smoking. Of the patients, 6 had been exposed to COVID-19: 4 came into contact with suspected or confirmed COVID-19 cases while hospitalized (30.8\%), and 2 were local residents of Wuhan
(15.4\%). Although the remaining patients had no clear epidemiological history, they were still exposed to a relevant COVID-19 environment, since they can move freely in Wuhan until Jan $23^{\text {rd }}$.

Four patients presented with fever without chills (30.8\%), but none had a high fever (defined as a body temperature greater than $39^{\circ} \mathrm{C}$ ). More than half complained of fatigue $(53.8 \% ; 7 / 13)$ and most had chest pain and shortness of breath due to their primary cardiovascular disease. Of the 13 patients, 7 had hypertension $(53.8 \%$ ) and 8 had taken ACE inhibitors (ACEI) or angiotensin receptor blockers (ARBs) prior to admission (61.5\%). Two patients had a history of heart surgery and 1 had a history of percutaneous coronary intervention (PCI) (Table S1).

\section{Preoperative imaging, laboratory findings, and COVID-19 diagnoses}

Due to the state of emergency and lack of standard COVID-19 PCR kits in the early phase of the pandemic, not all patients underwent chest CT or RT-PCR testing. Eleven patients received a chest CT scan; of these patients, 5 had multiple patchy ground-glass shadows detected in their lungs (Figure 1). Oral and pharyngeal swabs were taken from 4 patients and tested for SARS-CoV-2 using 
quantitative RT-PCR (qRT-PCR) kits (BioGerm, Shanghai, China) $(9,10)$. These 4 patients had negative PCR results. According to the $6^{\text {th }}$ edition of the Novel Coronavirus Pneumonia Prevention and Control Program published by the National Health Commission of China, 6 patients in this cohort were categorized as suspected cases (46.2\%). The remaining patients were excluded from diagnosis of COVID-19 (7/13; 53.8\%).

Laboratory tests showed that $69.2 \%(9 / 13)$ of these patients had abnormal white blood cell counts and 30.8\% (4/13) had lymphopenia. Less than half of these patients had abnormal cardiac enzyme results [AST, 38.5\%; LDH, 38.5\%; high sensitivity TNI (hsTNI), 22.2\%; CK, 9.1\%; CK-MB, 9.1\%]. Of this cohort, 5 patients had elevated concentrations of alanine aminotransferase $(38.5 \%)$ and 6 had increased levels of total bilirubin. Only part of the patients received tests for procalcitonin, brain natriuretic peptide (BNP) or N-terminal prohormone BNP (NTproBNP), and CRP [high sensitivity CRP (hsCRP)], and few of these patients received positive results (Table S2).

\section{Relevant surgical features and postoperative outcomes}

All 13 patients had undergone surgery as of 11 February 2020. The operations included 4 Sun's procedures [1 with concomitant coronary artery bypass grafting (CABG)], 3 congenital malformation corrections (1 being a repeated surgery), 2 heart transplants, 1 Bentall procedure with concomitant CABG, 1 off-pump CABG, 1 aortocoronary fistula repair (a repeated surgery), and 1 aortic valve replacement (AVR) with removal of paravalvular abscesses. Level 3 protection was used when surgery was performed on the 6 patients with suspected COVID-19. Level 2 protection was used for the 7 patients excluded from having COVID-19 (Table S3).

All patients were successfully extubated, with no tracheotomy, cardiopulmonary resuscitation, or IABP catheterization. No patients in this cohort died or developed postoperative severe acute respiratory syndrome (ARDS), renal failure, or septic shock. The primary postoperative complication was bacterial infection $(23.1 \% ; 3 / 13)$, all cases of which were Acinetobacter baumannii infections. Of the 13 patients, 1 developed postoperative right hemiplegia and 1 developed unilateral tension pneumothorax. Four patients had already been discharged within 28 days. All patients in this cohort were tested for SARS-CoV-2 after surgery and excluded from having COVID-19. No cross-infection occurred with other patients or medical staff close contacts.

\section{Discussion}

The COVID-19 pandemic began during the seasonal change from winter to spring. This period is also associated with a high incidence of acute and severe cardiovascular disease, such as aortic dissection and myocardial infarction. Some researchers have suggested a close relationship between COVID-19 and the cardiovascular system. The dyspnea, fever, and hypoxemia caused by pneumonia can affect the cardiovascular system and lead to cardiovascular diseases, including pulmonary hypertension, rightsided heart failure, and pulmonary embolism-associated cardiac arrest (11). The SARS-CoV-2 infection may also exacerbate preexisting cardiovascular disorders, particularly in older people and children, due to its high virulence and involvement in inflammation, downregulation of ACE2, and renin-angiotensin-aldosterone system (RAAS) activation. This infection also leads to hypertension, myocardial infarction, severe arrhythmia, and myocarditis $(1,6,12)$. Furthermore, although assisted mechanical ventilation and cardiopulmonary bypass are essential components of many cardiovascular operations, they have unfavorable effects on cardiopulmonary function, which presents another issue for the perioperative management of cardiovascular disease patients concomitant with COVID-19. Therefore, summaries of patient outcomes and the management of emergency cardiovascular surgeries during the COVID-19 pandemic are of great clinical significance.

In this study, we described the clinical data and outcomes of 13 patients who underwent emergency cardiovascular surgery at a sentinel hospital located at a COVID-19 epicenter; Wuhan Union Hospital is less than $5 \mathrm{~km}$ away from the source of the outbreak, the Huanan Seafood Wholesale Market. Following the pandemic outbreak, the first significant challenge cardiovascular surgeons faced was differential diagnoses, as the signs and symptoms of COVID-19 (such as fever, cough, shortness of breath, chest pain, and fatigue) are also common symptoms of cardiovascular disorders. A definitive diagnosis of COVID-19 still relies on chest CT imaging and qRTPCR $(13,14)$. However, in late January 2020, Wuhan doctors faced another problem: a shortage of PCR kits. Consequently, the average time to receive test results was longer than 24 hours. Patients in a critical condition that required immediate surgery could not wait to receive COVID-19 test results. Such critical conditions included the following: acute aortic dissection, acute myocardial 
infarction, complex congenital heart disease with severe hypoxia and circulation instability, congenital heart disease with severe pneumonia leading to severe pulmonary hypertension, uncontrolled heart failure with medication, cardiac tumor leading to hemodynamic instability, and other cardiovascular diseases associated with rapid deterioration and a high mortality rate. In situations where cardiovascular disease patients cannot wait for COVID-19 test results, surgeons, nurses, and anesthetists should adopt all integrated systemic protection measures as though a COVID-19 diagnosis has been confirmed.

Personal protective equipment (PPE) may hamper the performance of surgeons, particularly in delicate microsurgeries, such as off-pump CABG and arterial switch operations, as well as in well-established operations, such as aortic dissections. However, the surgical features of this cohort, such as operation time, CPB time, cross-clamp time, mechanical ventilation time, length of intensive care unit (ICU) stay, drainage time, and the need for blood transfusions, were similar to the surgical features of most patients in the normal period. Only a few patients in this cohort experienced postoperative complications, and none developed ARDS, renal failure, or septic shock, including the 2 heart transplant patients who were undergoing immunosuppressive therapy. No other patients or relevant medical staff were infected by SARS-CoV-2 within a period of at least 14 days of medical observation. As of 20 February 2020 , none of these patients had died or developed severe pneumonia.

The perioperative management of suspected and diagnosed COVID-19 cases at Wuhan Union Hospital are outlined as follows. (I) All suspected and diagnosed cases should be perioperatively isolated in a separate ICU. (II) Emergency surgery should be performed in a designated negative pressure operating room with a buffer area around it. (III) Antiviral agents (i.e., lopinavir, ritonavir, or arbidol) and/or immune modulators (interferon alpha) should be administered to all patients with suspected or diagnosed COVID-19. Empiric antibiotics are suitable to give to specified patients to prevent perioperative infection. (IV) Adequate support treatment should be given to maintain stable cardiopulmonary function. Patients with acute aortic dissection or unstable angina may be sedated to alleviate symptoms and reduce oxygen consumption. (V) Medical staff should correctly wear PPE, including goggles, N95 medical masks, waterproof boot covers, aseptic surgical clothing, and disposable aseptic protective gloves. All PPE should meet the requirements of level 3 protection. (VI) The use of protective lung ventilation strategies, such as low tidal volume ventilation, during invasive ventilation might be beneficial to patients. Closed endotracheal suctioning systems and airtight ventilator circuits should be used to prevent virus transmission. (VII) Reservation of the central venous catheter is recommended in order to monitor patients' postoperative central venous pressure (CVP), maintain blood volume balance and hemodynamic stability, and to avoid exacerbating pulmonary exudates or worsening hypoxia. (VIII) Special attention should be paid to avoid droplet transmission, as well as any contact with respiratory secretions and body fluids that may occur during procedures such as surgeries, endotracheal intubations, sputum suctioning, recruitment maneuvers, non-invasive ventilation, tracheotomies, cardiopulmonary resuscitation, bronchoscopies, intra-aortic balloon pump (IABP) catheterization, and extracorporeal membrane oxygenation (ECMO) catheterization. (IX) Assuming they do not violate medical principles, simple yet effective surgical strategies and techniques are preferred to minimize patient trauma, as well as the risk of cross-infection. These perioperative guidelines are summarized in Figure 2.

\section{Limitations}

This study was limited by its small sample size and retrospective nature. Several points should be considered when interpreting these findings. Firstly, no patient in this cohort returned a positive laboratory test, so we were unable to determine what effect COVID-19 might have on cardiovascular surgery. The high false negative rate of the qRT-PCR test also raises some concerns; certain levels of misdiagnosis would impact some of our findings.

\section{Conclusions}

This study demonstrated that emergency cardiovascular surgery can be safely provided to patients with acute and severe cardiovascular disease during the COVID-19 pandemic. Adequate preparation and provision of appropriate treatment are the most pivotal issues to address in order to ensure satisfactory patient outcomes. 


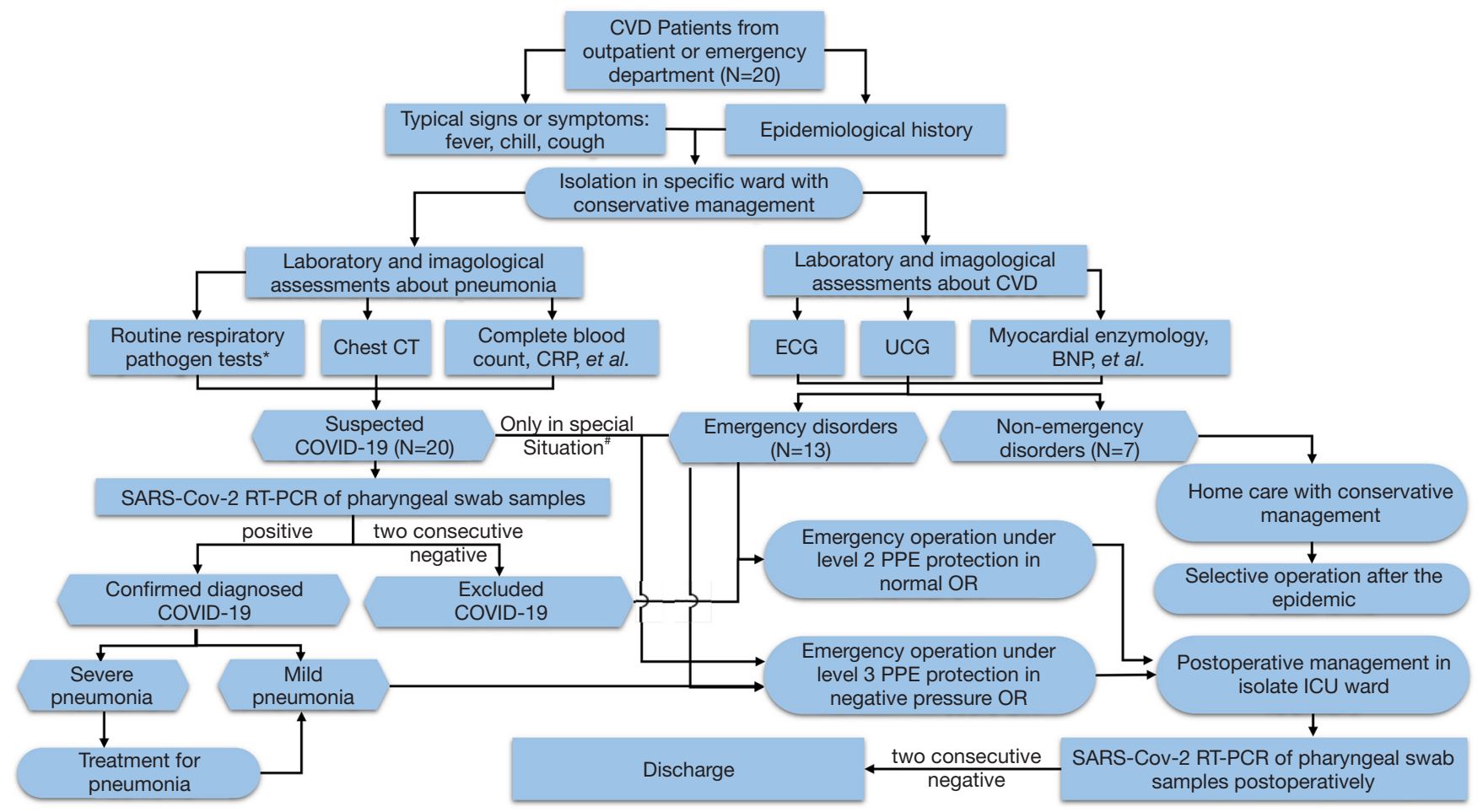

Figure 2 Flow chart for the management of emergency cardiovascular surgery during the COVID-19 pandemic. BNP, brain natriuretic peptide; CRP, C-reactive protein; CT, computed tomography; CVD, cardiovascular disease; PPE, personal protective equipment; SARS$\mathrm{CoV}-2$, severe acute respiratory syndrome coronavirus 2. *, inclusive of influenza type A and B, respiratory syncytial virus, coxsackie group B virus, adenovirus, mycoplasma pneumonia, and chlamydia pneumonia. ", in the early phase of the pandemic, due to a lack of PCR kits, some emergency suspected COVID-19 cases required cardiovascular long surgery before a diagnosis was confirmed. These were classified as "clinically diagnosed cases" according to the $5^{\text {th }}$ edition of the Novel Coronavirus Pneumonia Prevention and Control Program.

\section{Acknowledgments}

Funding: This study was financially supported by the National Natural Science Foundation of China Young Scientists Fund (No. 81400290) to XH.

\section{Footnote}

Reporting Checklist: The authors have completed the STROBE reporting checklist. Available at http://dx.doi. org/10.21037/jtd-20-1649

Data Sharing Statement: Available at http://dx.doi. org/10.21037/jtd-20-1649

Conflicts of Interest: All authors have completed the ICMJE uniform disclosure form (available at http://dx.doi. org/10.21037/jtd-20-1649). The authors have no conflicts of interest to declare.

Ethical Statement: The authors are accountable for all aspects of the work in ensuring that questions related to the accuracy or integrity of any part of the work are appropriately investigated and resolved. This study was conducted in accordance with the Declaration of Helsinki (as revised in 2013), and approved by the Medical Ethical Committee of Wuhan Union Hospital of Huazhong University of Science and Technology \{[2020]IRS(S229)\}. Written informed consent was obtained from all study participants or their legal guardians; patient anonymity was assured throughout the research process.

Open Access Statement: This is an Open Access article distributed in accordance with the Creative Commons Attribution-NonCommercial-NoDerivs 4.0 International License (CC BY-NC-ND 4.0), which permits the non- 
commercial replication and distribution of the article with the strict proviso that no changes or edits are made and the original work is properly cited (including links to both the formal publication through the relevant DOI and the license). See: https://creativecommons.org/licenses/by-nc-nd/4.0/.

\section{References}

1. Huang C, Wang Y, Li X, et al. Clinical features of patients with 2019 novel coronavirus in Wuhan, China. Lancet 2020;395:497-506.

2. Gorbalenya AE, Baker SC, Baric RS, et al. Severe acute respiratory syndrome-related coronavirus: The species and its viruses, a statement of the Coronavirus Study Group. BioRxiv 2020.02.07.937862. Available online: https://doi. org/10.1101/2020.02.07.937862

3. WHO main website. Available online: https://www.who. int. Accessed February 26, 2020.

4. Website of National Health Commission of the People's Republic of China. Available online: http://www.nhc.gov. cn. Accessed on February 26, 2020.

5. Zhou P, Yang XL, Wang XG, et al. A pneumonia outbreak associated with a new coronavirus of probable bat origin. Nature 2020;579:270-3.

6. Zhao Y, Zhao ZX, Wang YJ, et al. Single-cell RNA expression profiling of ACE2, the putative receptor of Wuhan 2019-nCov. BioRxiv 2020.01.26.919985. Available online: https://doi.org/10.1101/2020.01.26.919985

7. WHO. Clinical management of severe acute respiratory infection when novel coronavirus $(\mathrm{nCoV})$ infection is suspected. Interim guidance. Available online: https://

Cite this article as: Hu X, Wang Y, Xia J, Dong N, Shi J. Managements of 13 emergency cardiac surgeries under COVID-19 pandemic in a Sentinel Hospital. J Thorac Dis 2020;12(11):6663-6669. doi: 10.21037/jtd-20-1649 www.who.int/docs/default-source/coronaviruse/clinicalmanagement-of-novel-cov.pdf. Accessed January 12, 2020.

8. National Health Commission of China. New Coronavirus Pneumonia Prevention and Control Program (5th revision edition). Available online: http://www.gov.cn/zhengce/ zhengceku/2020-02/09/5476407/files/765d1e65b7d14430 81053c29ad37fb07.pdf. Accessed February 23, 2020.

9. WHO. Laboratory testing for 2019 novel coronavirus (2019-nCoV) in suspected human cases. Interim guidance. Available online: https://www.who.int/publicationsdetail/laboratory-testing-for-2019-novel-coronavirus-insuspected-human-cases-20200117. Accessed January 17, 2020.

10. Corman VM, Landt O, Kaiser M, et al. Detection of 2019 novel coronavirus (2019-nCoV) by real-time RT-PCR. Euro Surveill 2020;25:2000045.

11. Corrales-Medina VF, Musher DM, Shachkina S, et al. Acute pneumonia and the cardiovascular system. Lancet 2013;381:496-505.

12. Sun $\mathrm{P}, \mathrm{Lu} \mathrm{X}, \mathrm{Xu} \mathrm{C}$, et al. Understanding of COVID-19 based on current evidence. J Med Virol 2020;92:548-51.

13. Zhang J, Zhou L, Yang Y, et al. Therapeutic and triage strategies for 2019 novel coronavirus disease in fever clinics. Lancet Respir Med 2020;8:e11-2.

14. Wang Y, Kang H, Liu X, et al. Combination of RT-qPCR Testing and Clinical Features for Diagnosis of COVID-19 facilitates management of SARS-CoV-2 Outbreak. J Med Virol 2020;92:538-9.

(English Language Editors: B. Madden and J. Reynolds) 